\title{
EUSO-Balloon mission to record extensive air showers from near space
}

\author{
Lawrence Wiencke* \\ JEM-EUSO Collaboration \\ E-mail: lwiencke@mines.edu
}

The first EUSO-Balloon stratospheric flight recorded artificial tracks and pulses that were generated using a laser and optical flashers flown in helicopter under the balloon. The time at float altitude was 5 hours. The balloon was launched from Timmins, Ontario by the French Space Agency, CNES and the helicopter underflight was supported by NASA. To make the first measurements of high energy cosmic ray extensive air showers from near space, preparations are underway for a super pressure balloon flight of several weeks duration. The proposed launch site is Wanaka, New Zealand. We describe the parameters of this mission, the updated instrument, and the expected rates of extensive air showers events produced by cosmic primaries in the energy range of $5 \times 10^{17} \mathrm{eV}$ to $2 \times 10^{19} \mathrm{eV}$. A precursor short duration flight from Aire sur l'Adour, France is also envisioned to test improvements to the instrument.

The 34th International Cosmic Ray Conference,

30 July- 6 August, 2015

The Hague, The The Hague

*Speaker. 


\section{Introduction}

The origin of the highest energy subatomic particles known to exist in the universe remains an open question as do the acceleration mechanisms of the sources. Most of what is known about the universe beyond our solar system has been obtained by measuring photons. Yet extreme energy cosmic rays (EECRs) have been measured with energies beyond $100 \mathrm{EeV}$. This energy exceeds the highest energy photon measurements by more than 6 orders of magnitude. EECRs offer a window into the extreme universe. The measured energy spectrum of EECRs is non-thermal. The flux falls well below the rate of one particle $/ \mathrm{km}^{2} /$ century at $100 \mathrm{EeV}$.

The challenge to realize a detector aperture that is an order of magnitude larger than that of ground based detectors and then to map the entire EECR sky with this single instrument motivates the Extreme Universe Space Observatory on board the Japanese Experimental Module (JEMEUSO). JEM-EUSO will look down on the earth from the International Space Station (ISS). Using refractive optics to obtain a $+/-30^{\circ}$ field of view (FoV), JEM-EUSO will view an observational area of $1.4 \times 10^{5} \mathrm{~km}^{2}$ [1]. The detector will image the traces of UV light from extensive air showers (EAS) produced by the interaction of EECRs in the atmosphere. This unique instrument will also have sensitivity to other UV signatures in the atmosphere that range from atmospheric transient luminous events, to meteoroids, to photons and neutrinos, to exotic objects, for example strange quark matter.

The EUSO super pressure balloon (EUSO-SPB) experiment will be the first to measure EASs produced by EECRs from above. The proposed launch site is Wanaka NZ. For reference, the path of NASA's first super pressure balloon flight that was launched from this site (March 2015) is shown in figure 1 below. Flying the EUSO-SPB instrument on an ultra-long duration balloon flight of at least 20 nights centered on the new moon will provide the exposure that is required to achieve this important milestone. This mission will be the first extended duration operation of a cosmic ray UV fluorescence detector at near space altitude and provide an important end-to-end test of JEM-EUSO. The EUSO-SPB mission will also afford the possibility to detect other faint and fast UV light flashes that could be a background for EAS observations or be of interest in their own right with some discovery potential.

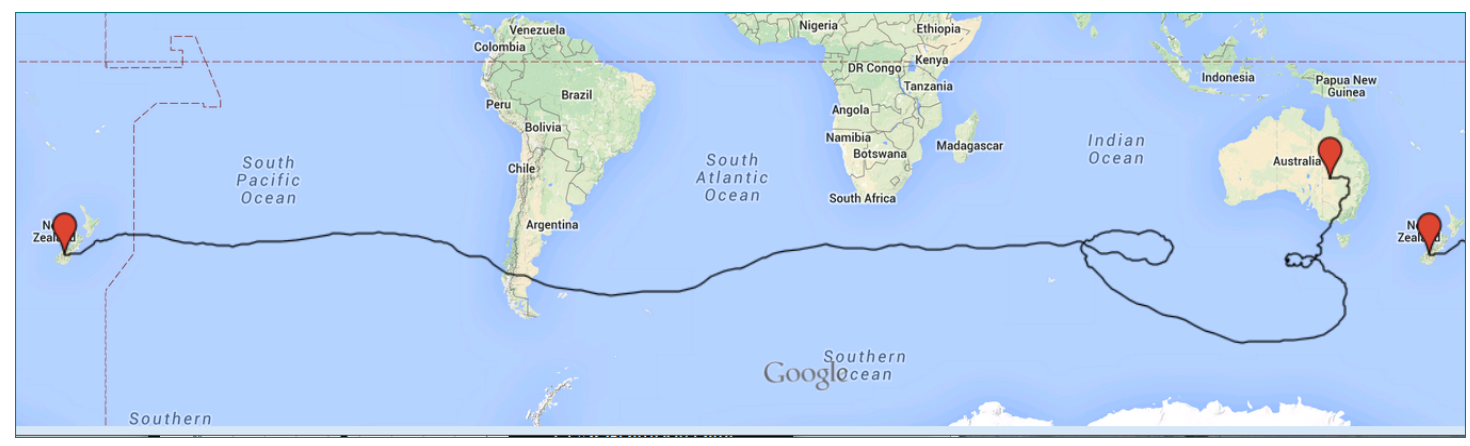

Figure 1: The path of the 2015 NASA Super Pressure Balloon engineering flight launched from Wanaka NZ. The flight duration was 32 days.

The EUSO-SPB instrument will image light from the nitrogen fluorescence and Cherenkov light created by the EASs. The UV images of EASs and other triggered events will be captured by 
a 2304 pixel high-speed camera with a time resolution of $2.5 \mu \mathrm{s}$. The "video clips" recorded will show the trajectories of EASs as they develop in the atmosphere. Near the start of the flight the instrument will also record simulated EAS signals from a calibrated LED+UV laser system flown on an aircraft within the FoV of EUSO-SPB.

\section{The EUSO-SPB Instrument}

The EUSO-SPB instrument is an updated version of the first EUSO-Balloon instrument (Fig. 2) that was flown successfully from the Timmins Stratospheric Balloon Facility on August 24/25 2015. During the Timmins mission, the EUSO-Balloon instrument recorded data for 5 hours at a float altitude of $38 \mathrm{~km}$, measured UV backgrounds over different landscapes [4] and recorded IR images [5] of the FoV. It also recorded 270 sequences of UV flashes [6] and tracks [7] that were generated by three light sources, including a pulsed laser, that were flown under the balloon in a helicopter [8]. EUSO-SPB will build on the success and experience of this mission.

Details of EUSO-Balloon including the hardware, Timmins mission, and preliminary results are presented in references [2] and [3]. Briefly, the instrument is a $1 \mathrm{~m} \mathrm{UV}$ telescope with refractive optics and a $12^{\circ} \times 12^{\circ} \mathrm{FoV}$. The focal surface is instrumented with a photon detector module (PDM) that uses the design and components that are planned for the 137 PDM focal surface of JEM-EUSO. The PDM is covered with a UV filter assembly that passes light between $290 \mathrm{~nm}$ and $430 \mathrm{~nm}$ to span the UV fluorescence spectrum of EASs. The PDM is divided into 2304 pixels, each with a FoV of $0.25^{\circ} \times 0.25^{\circ}$. In comparison, 9 HiRes fluorescence detector telescopes [9] contain a total of 2304 pixels. The 2304 pixel ouputs are digitized in $2.5 \mu$ s bins. The system is operated in single
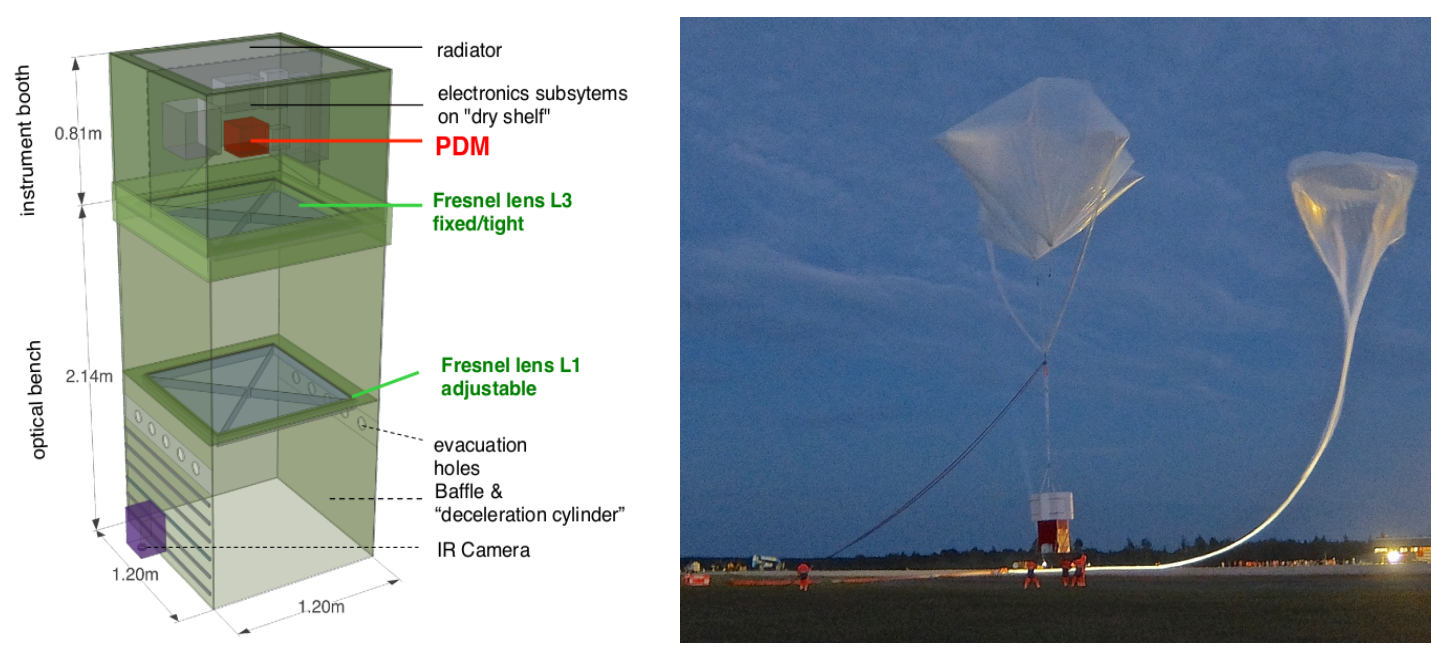

Figure 2: Left: The EUSO-Balloon instrument as flown in 2014. Light entered the open bottom end of the gondola box and was focused by two fresnel lenses on to the photon detector module (PDM). Right: The instrument at the Timmins Canada launch site. The white structure surrounding the upper half of the payload provided floatation in water. (The instrument happened to land in a small lake.). The two balloons directly above lifted the payload off the ground. The stratospheric balloon seen ascending on the far right carried the payload up to float altitude for the overnight data collection mission. 
photoelectron (PE) counting mode. An event contains the digitized PE counts of all pixels for 128 consecutive time bins. This corresponds to a readout window of $320 \mu$ s duration.

The EUSO-SPB instrument will included the upgrades listed below.

\begin{aligned} Item & Description \\ \hline MAPMTs & $\begin{array}{l}\text { Higher quantum efficiency multi-anode photomultiplier tubes } \\ \text { (Hamamatsu model R11265-113-M65 MOD2) }\end{array} \\$\hline Solar Power & $\begin{array}{l}\text { Solar Panels and updated power system. Panels will be mounted } \\ \text { on the 4 sides of the gondola for redundancy. }\end{array} \\$\hline Optics & $\begin{array}{l}\text { Include a third fresnel lens that was in the original design to pro- } \\ \text { vide chromatic correction over the EAS UV spectrum. }\end{array} \\$\hline HV & Updated HV distribution system \\ \hline Trigger & Upgraded trigger for EASs \\ \hline Control & Update system control programming for a 50 day mission \\ \hline Telemetry & $\begin{array}{l}\text { Interface to the Columbia Scientific Balloon Facility's Support } \\ \text { Instrument Package (SIP) }\end{array}\end{aligned}$

\section{Estimating the EAS event rate}

A Monte Carlo simulation analysis was performed to estimate the number and the energy range of EASs that EUSO-SPB will record [10]. A version of the CONEX [11] EAS simulation package was modified for use with a volume detector. Sets of 60,000 proton induced EASs were simulated between $0^{\circ}$ and $80^{\circ}$ in zenith angle. Using the JEM-EUSO Offline software framework [12], the EASs were randomized in azimuth angle and core location over a $150 \mathrm{~km}$ radius circle (Fig. 3).

Eight simulated data sets were generated and randomized in this manner corresponding to 8 energy steps from $10^{17.75} \mathrm{eV}$ to $10^{19.25} \mathrm{eV}$. For each EAS in each set, the UV optical emission in the atmosphere was then simulated. The EASs for which a minimum number of UV scintillation
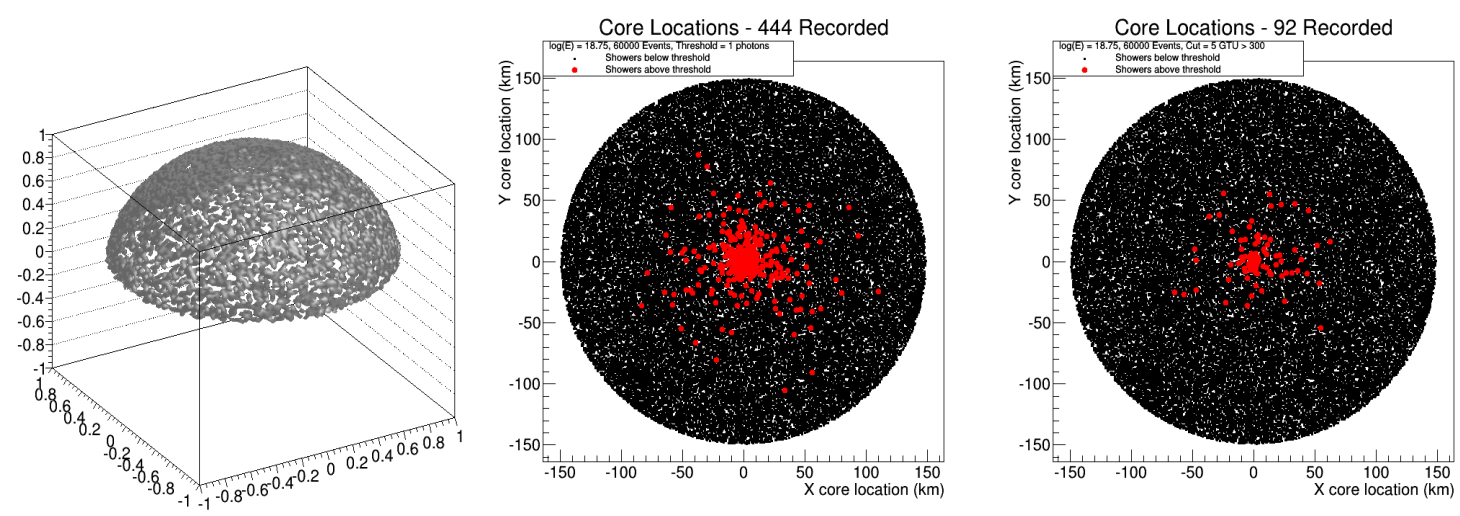

Figure 3: Left: Sample distribution of simulated EAS directions projected on to a unit hemisphere. Center: EAS core location projected to the ground. Black points indicate all the events simulated. Red points indicated events for which some light entered the EUSO-SPB optical aperture. Right: Similar to the adjacent plot with the additional requirement that at least 300 photons reach EUSO-SPB on at least each of 5 consecutive $2.5 \mu$ s time bins. The energies of these simulated events is $10^{18.75} \mathrm{eV}$. 
photons reached the optical aperture (reached lens within the FoV) of EUSO-SPB were identified. A further selection required a minimum number of photons in each of 5 consecutive time bins. An example event track and light curve are displayed in figure 4 with selection cut minium of 300 photons. For an overall efficiency factor of 0.40 (optics transmission) x 0.25 (quantum efficiency) x 0.25 (fraction of point spread function on one pixel), this corresponds to at least 7.5 PEs per time bin on the brightest pixels. This level is expected to be well above the UV background rates that are expected to be about $1 \mathrm{PE}$ per pixel per time bin. About half of these events have the depth of shower maximum in the field of view bracketed by at least two time bins. Further examples are shown in reference [13].

The EAS detection rate was calculated as $N=\int J(E) A_{s i m} f(E) d E$ where $\mathrm{N}$ is the number of EASs detected per unit time, $\mathrm{J}(\mathrm{E})$ is the measured cosmic ray flux as a function of energy $\mathrm{E}$ as reported in [14], $A_{\text {sim }}$ is the simulated aperture of the $150 \mathrm{~km}$ radius circle over the $0^{\circ}$ to $80^{\circ}$ zenith angle range $\left(A_{s i m}=3.7 \times 10^{5} \mathrm{~km}^{2} \mathrm{sr}\right)$, and $\mathrm{f}(\mathrm{E})$ is fraction, for a particular energy, of the simulated EASs that would be observed by EUSO-SPB. The simulated $\mathrm{f}(\mathrm{E})$ data and the product $\mathrm{J}(\mathrm{E}) A_{\text {sim }} \mathrm{f}(\mathrm{E})$ are plotted in figure 5 for various photon minima. The estimated aperture, $A_{\text {sim }} \mathrm{f}\left(\mathrm{E}=10^{19.25} \mathrm{eV}\right)$ approaches $1000 \mathrm{~km}^{2} \mathrm{sr}$ for EASs that expected to be clearly observable. The minimum energy is estimated to be about $10^{18.25} \mathrm{eV}$. The detection rates for three different photon thresholds are listed in table 1 . The nominal rate is about 0.2 per hour. This estimate is consistent with the results of a more detailed study [15] peformed with the ESAF [16] simulation framework.
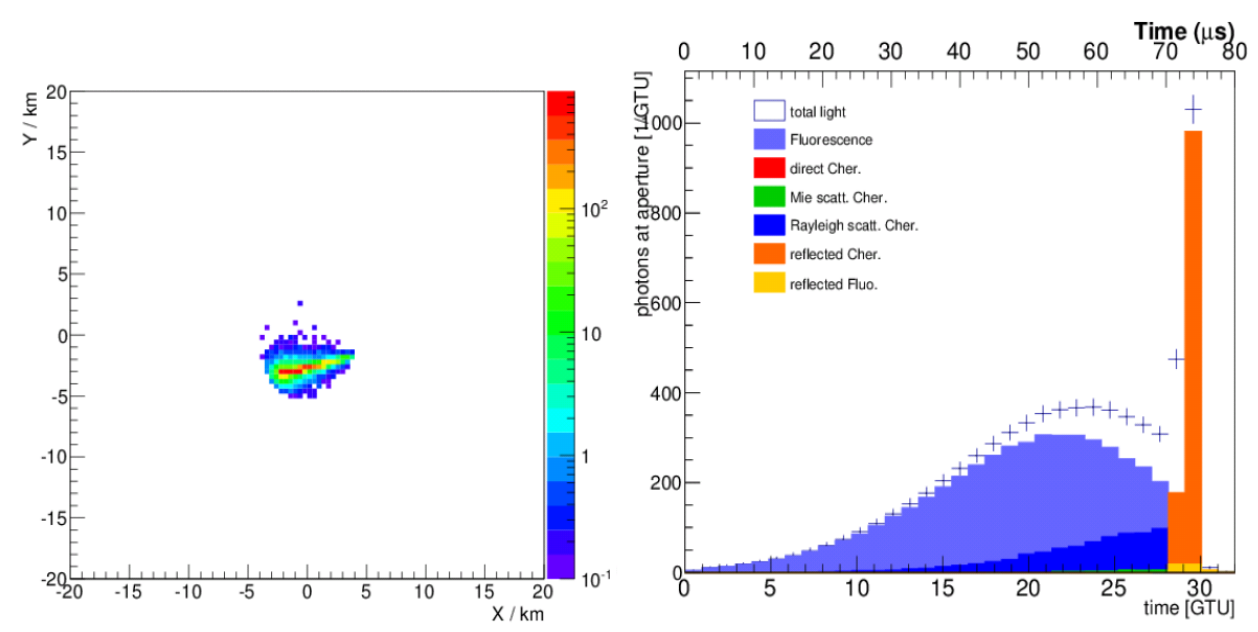

Figure 4: An example simulated event $\left(\mathrm{E}=10^{18.75} \mathrm{eV}\right)$ passing the selection criteria for EUSO-SPB (see text). Left: The projected track Right: The EAS light curve

\section{Notes about the EUSO-SPB Mission}

The proposed launch date for EUSO-SPB is March 2017. The 2017 March/April dark period at the latitude of Wanaka, NZ includes18 nights with at least 2.5 hours of moon down dark time for a total of 118 hours (Table 2). The average moon down dark time per night is 6.5 hours. The start times and end times and, to a lesser extent, the total amount of darkness will vary depending on the trajectory of the balloon. In practice, the dark times will be reduced slightly because a net eastward 

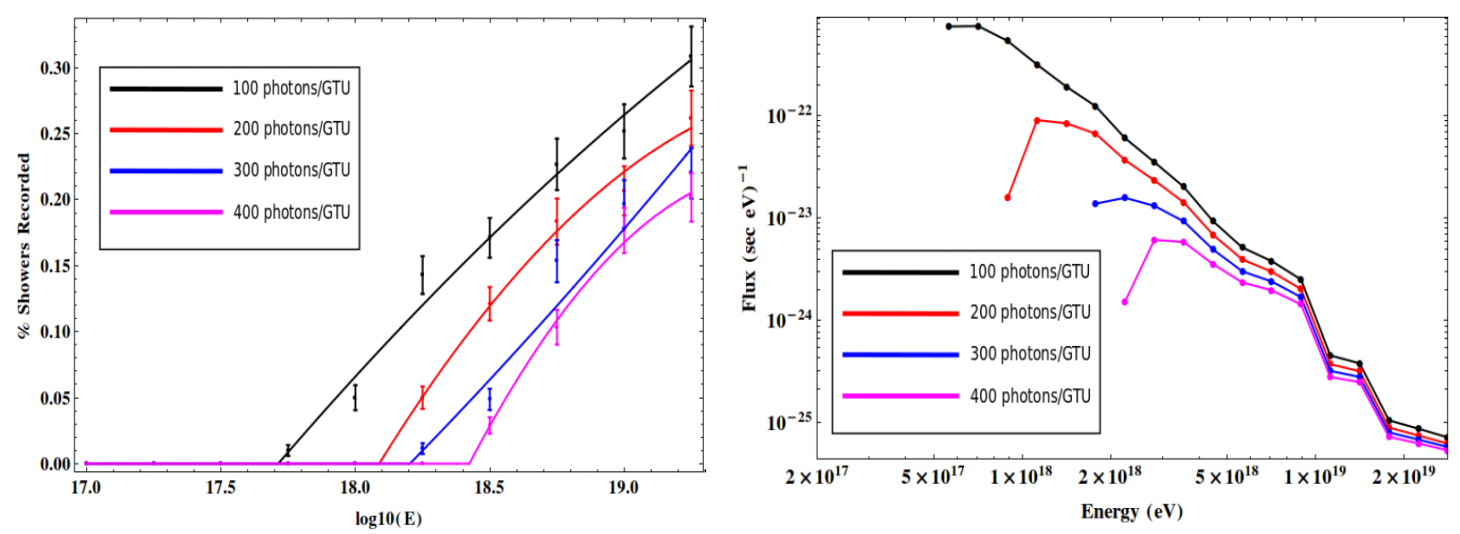

Figure 5: Left: The fraction of observable EASs relative the the number simulated for different photon thresholds (see text). Right: The flux of EASs observable by EUSO-SPB. The integral of these curves yields the expected event rate.

\begin{tabular}{|lll|}
\hline $\begin{array}{l}\text { Photon } \\
\text { Threshold }\end{array}$ & $\begin{array}{l}\text { Events/ } \\
\text { hour }\end{array}$ & $\begin{array}{l}\text { Events/ } \\
\text { dark period }\end{array}$ \\
\hline 200 & 0.42 & 50 \\
300 & 0.18 & 21 \\
400 & 0.09 & 11 \\
\hline
\end{tabular}

Table 1: Expected Cosmic Ray Air Shower detection rates. Photon threshold is the number of photons reaching the instrument optical enterance aperture in each of 5 consecutive $2.5 \mu$ s timebins. The dark period assumed is listed in table 2.

drift of the balloon will lead to slightly earlier moonrise and sunrise times. This reduction may be offset by collecting additional data during low moon periods.

The expected data telemetry transfer rate from the balloon to the ground is $180 \mathrm{kbits} / \mathrm{s}$ using two Tracking and Data Relay Satellite System (TDRSS) data links. On nights with the longest dark period $(9 \mathrm{~h})$, the data collected at an average event rate of $0.25 \mathrm{~Hz}$ could be downloaded over a 24 hour period. A separate Irridium satellite link will provide a low rate channel for sending simple commands and queries to the instrument control computer.

At the start of the mission a UV LED and a pulsed $355 \mathrm{~nm}$ laser will be flown in an aircraft under the balloon for several hours to provide a set of calibration flashes and tracks for the instrument. The aircraft will fly below $10,000 \mathrm{ft}$ so that the UV laser beam can be fired out an open window. The intention is to perform these tests while there is still line of site communications to the balloon so that data can be downloaded at a reasonably high rate.

\section{Conclusion}

EUSO-SPB is a proposed super pressure long duration balloon experiment to make the first observation and measurement of cosmic ray extensive air showers by looking down from near space with a fluorescence detector. The instrument is an updated version of the detector flown successfully in 2014 that recorded the first (laser) tracks by looking down. For EUSO-SPB, the 
number of obvious EAS events expected is about 10-15 for a 20 night mission that spans a dark period. The instrument will also record UV backgrounds over the ocean and record faint pulses that may be a background to the EAS signature or may be of interest by their own right. The discovery potential of this mission to observe fast UV optical signatures of previously unmeasured phenonena can not be excluded.

\begin{tabular}{|l|l|ll|}
\hline date UTC & hrs dark & start UTC & stop UTC \\
\hline 2017 Mar 19 & $02: 08$ & $08: 38$ (twilight) & $10: 46$ (moon 62\%) \\
2017 Mar 20 & $02: 52 *$ & $08: 36$ (twilight) & $11: 28$ (moon 52\%) \\
2017 Mar 21 & $03: 40 *$ & $08: 34$ (twilight) & $12: 15$ (moon 42\%) \\
2017 Mar 22 & $04: 34 *$ & $08: 32$ (twilight) & $13: 07$ (moon 32\%) \\
2017 Mar 23 & $05: 34 *$ & $08: 30$ (twilight) & $14: 04$ (moon 23\%) \\
2017 Mar 24 & $06: 38 *$ & $08: 28$ (twilight) & $15: 06$ (moon 14\%) \\
2017 Mar 25 & $07: 46 *$ & $08: 26$ (twilight) & $16: 12$ (moon 7\%) \\
2017 Mar 26 & $08: 51 *$ & $08: 24$ (twilight) & $17: 15$ (twilight) \\
2017 Mar 27 & $08: 54 *$ & $08: 22$ (twilight) & $17: 16$ (twilight) \\
2017 Mar 28 & $08: 57 *$ & $08: 20$ (twilight) & $17: 18$ (twilight) \\
2017 Mar 29 & $09: 01 *$ & $08: 18$ (twilight) & $17: 19$ (twilight) \\
2017 Mar 30 & $09: 04 *$ & $08: 16$ (twilight) & $17: 20$ (twilight) \\
2017 Mar 31 & $08: 40 *$ & $08: 41$ (moon 14\%) & $17: 22$ (twilight) \\
2017 Apr 1 & $07: 57 *$ & $09: 25$ (moon 24\%) & $17: 23$ (twilight) \\
2017 Apr 2 & $07: 08 *$ & $10: 16$ (moon 35\%) & $17: 24$ (twilight) \\
2017 Apr 3 & $06: 12 *$ & $11: 13$ (moon 46\%) & $17: 25$ (twilight) \\
2017 Apr 4 & $05: 12 *$ & $12: 14$ (moon 58\%) & $17: 27$ (twilight) \\
2017 Apr 5 & $04: 09 *$ & $13: 19$ (moon 69\%) & $17: 28$ (twilight) \\
2017 Apr 6 & $03: 04 *$ & $14: 24$ (moon 79\%) & $17: 29$ (twilight) \\
2017 Apr 7 & $02: 00$ & $15: 30$ (moon 87\%) & $17: 31$ (twilight) \\
\hline
\end{tabular}

Table 2: Example of expected hours of operation for a dark period at $45^{\circ} \mathrm{S}$ (see text). This dark period includes 18 days, indicated by an asterix, with at least 2.5 hours of moon down darkness for a total of 118 hours. These hours would be the primary targeted observation times for EUSO-SPB.

Acknowledgment: This work was partially supported by Basic Science Interdisciplinary Research Projects of RIKEN and JSPS KAKENHI Grant (22340063, 23340081, and 24244042), by the Italian Ministry of Foreign Affairs, General Direction for the Cultural Promotion and Cooperation, by the 'Helmholtz Alliance for Astroparticle Physics HAP' funded by the Initiative and Networking Fund of the Helmholtz Association, Germany, and by Slovak Academy of Sciences MVTS JEM-EUSO as well as VEGA grant agency project 2/0076/13. Russia is supported by the Russian Foundation for Basic Research Grant No 13-02-12175-ofi-m. The US is supported by the NASA grants NNX13AH55G, NNX13AH53G. The Spanish Consortium involved in the JEM-EUSO Space Mission is funded by MICINN \& MINECO under the Space Program projects: AYA2009-06037-E/AYA, AYA-ESP2010-19082, AYA-ESP2011-29489-C03, AYAESP2012-39115-C03, AYA-ESP2013-47816-C4, MINECO/FEDER-UNAH13-4E-2741, CSD2009-00064 (Consolider MULTIDARK) and by Comunidad de Madrid (CAM) under projects S2009/ESP-1496 \& S2013/ICE-2822.

\section{References}

[1] The JEM-EUSO Collaboration, J.H. Adams et al., An evaluation of the exposure in nadir observation of the JEM-EUSO mission, Astroparticle Physics 44, 76-90, (2013). (arXiv:1305.2478) 
[2] P. von Ballmoos et al., (JEM-EUSO collaboration), General overview of EUSO-balloon mission in Proc. 34th ICRC, (The Hague), (2015).

[3] M. Bertaina et al., (JEM-EUSO collaboration), Preliminary results from the EUSO-Balloon flight in Proc. 34th ICRC, (The Hague), (2015).

[4] S. Mackovjak et al., (JEM-EUSO collaboration), Night Time Measurement of the UV Background by EUSO-Balloon in Proc. 34th ICRC, (The Hague), (2015).

[5] M. Frias et al., (JEM-EUSO collaboration), The infrared camera onboard the EUSO-balloon flight on August 24, 2015 in Proc. 34th ICRC, (The Hague), (2015).

[6] J. Adams et al., (JEM-EUSO collaboration), The calibration of EUSO-balloon using airborne light sources mounted to a helicopter in Proc. 34th ICRC, (The Hague), (2015).

[7] J. Eser et al., (JEM-EUSO collaboration), EUSO-Balloon: Observation and Measurement of Tracks from a laser in a Helicopter in Proc. 34th ICRC, (The Hague), (2015).

[8] D. Weiner. Cosmic-Ray Telescope Flies High, Scientific American, Vol 311, No. 5 (2014). (http://www.scientificamerican.com/article/cosmic-ray-telescope-flies-high/)

[9] T. Abu-Zayyad et. al., The prototype high-resolution Fly's Eye Detector cosmic ray detector NIMA, 450(2): 253-269, (2000).

[10] J. S. Fenn, Estimating the cosmic ray extensive air shower detection rate for the EUSO-Super Pressure Balloon mission, MSc Dissertation, Colorado School of Mines (2015). (http://hdl.handle.net/11124/17150)

[11] T. Bergmann et al., One-dimensional Hybrid Approach to Extensive Air Shower Simulation. Astropart. Phys., 26:420-432, (2007).

[12] T. Paul et al., (JEM-EUSO collaboration), A new design for simulation and reconstruction software for the JEM-EUSO mission in Proc. 34th ICRC, (The Hague), (2015).

[13] F. Fenu et al., (JEM-EUSO collaboration), The Simulation of cosmic rays in EUSO-Balloon: performance in the direction and energy reconstruction in Proc. 34th ICRC, (The Hague), (2015).

[14] The Pierre Auger Collaboration, J. Abraham et al., Measurement of the Energy Spectrum of Cosmic Rays Above $10^{18} \mathrm{eV}$ using the Pierre Auger Observatory, Physics Letters B, 685(45):239 - 246, (2010). (arXiv:1002.1975 [astro-ph.HE])

[15] S. Bacholle et al., (JEM-EUSO collaboration), EUSO-Balloon trigger efficiency in preparation of a long duration flight in Proc. 34th ICRC, (The Hague), (2015).

[16] F. Fenu et al., (JEM-EUSO collaboration), The ESAF simulation framework for the JEM-EUSO mission in Proc. 32rd ICRC, (Beijing), Vol 3, \#0592 (2011). (DOI: 10.7529/ICRC 2011/V03/0592) 